\title{
SPEED TRAJECTORY OPTIMIZATION FOR A HIGH-SPEED TRAIN
}

\author{
ZHUANG XIAO, ZINING ZHAO, QINGYUAN WANG, PENGFEI SUN \& XIAOYUN FENG \\ School of Electrical Engineering, Southwest Jiaotong University, People's Republic of China
}

\begin{abstract}
The high-speed railway system has been a significant energy consumer due to the rapid increase of operation mileage and speed. Eco-driving is a promising method to reduce energy consumption, which aims at calculating speed profiles with minimization electric energy consumption under temporal and spatial operation constraints. Cruise control is regarded as the most energy-efficient control strategy when the cost function is the mechanical energy on wheels or the efficiency of traction drive system is simplified as a constant value. However, the efficiency of the traction drive system is varies among different traction force and speed pairs. This paper presents an eco-driving cycle computation problem for high-speed trains considering nonlinear loss of traction drive system. On basis of this model, the "traction-and-coasting" driving strategy is proposed to replace the cruise mode, and corresponding bi-level optimization algorithm is designed. The energy saving performance is investigated by comparing optimized results with the solution based on cruise control. Numerical simulation results illustrate that the traction energy consumption can be reduced by the proposed strategy, and the operation efficiency of the traction drive system can be improved. The economy significance of this method is illustrated and analyzed. For application, the proposed method can be used for a driver assistant system (DAS) or an automatic train operation (ATO) system, which can significantly improve the energy economy of the high-speed railway.

Keywords: high-speed railway, eco-driving, energy calculation, driver advisory system.
\end{abstract}

\section{INTRODUCTION}

The energy consumption of high-speed trains (HSTs) is increasing rapidly, which has become a significant consumer in transportation sector [1]. Most HSTs have adaptive cruise control system, which can help drivers to maintain a constant speed on the track without steep hills. Although the cruise mode can reduce the energy consumption caused by the basic resistance [2], the load of the traction transmission system (TTS) is low in most cases, which might result in low efficiency of the TTS. On basis of that, the electrical energy from the traction power system is not the smallest [3]. To reduce energy consumption of the HSTs from the catenary and improve efficiency of the TTS, a new optimization algorithm to generate energy-efficient strategies should be carried out.

Various studies about calculating energy saving speed profiles have already been applied to railway vehicles [4], [5]. These methods can be categorized into two groups:

1. Different numerical search algorithms based on discrete state space are widely used to solve the energy saving optimization problem directly.

2. The construction of speed profile is achieved by the linkage of the optimal modes that are derived from the maximum principle.

For the first kind of method, an nonlinear programming model is established according to the operation constraints such as speed limitations, trip time and traction forces, and then different algorithms are applied to obtain the eco-driving trajectory: brute force search [6], Dynamic Programming [7], Genetic Algorithms [8] and pseudo-spectral method [9]. With the increase of operation speed and distance between two stations, the search space would increase exponentially, and the computation burden would increase significantly. In addition, 
the HSTs are driven by drivers manually. The frequent changing of operation modes would be infeasible to guide drivers. The second kind of method can avoid this problem due to specific operation modes. The summary of the switching principle among different optimal modes is presented in [10], [11]. An effective dual-loop algorithm is applied to locate the switching position and the sequence of different optimal modes [12].

The above studies aim at obtaining minimum mechanical energy on wheels, and the nonlinear power loss of the traction transmission system (TTS) is ignored [13]. The low load of the TTS would result in the low energy efficiency of the TTS when the train is in cruise mode. The maximum traction-coasting pair to replace cruise mode can improve the system efficiency in some situations.

This paper presents a bi-level optimization method that decreases energy consumption and improves energy efficiency. Specifically, the speed profile with the minimum mechanical energy is calculated first based on the Pontryagin Maximum Principle (PMP), and then traction-coasting pairs are used to replace cruise mode when the energy saving can be achieved. In addition, the quantitative analysis about the factors on energy efficiency of the traction-coasting pairs is discussed.

This paper is organized as follows. The system model and problem statement are described in Section 2. Section 3 illustrates the optimization method. The quantitative analysis of the energy efficiency of the traction-coasting pair is presented in Section 4. Case studies are carried out in Section 5.

\section{SYSTEM MODEL AND PROBLEM STATEMENT}

The acceleration/deceleration of the HST is controlled by the mechanical power on wheels. The electric energy is provided from the catenary. The energy conversion between electric and mechanical energy is achieved by the TTS, and the efficiency of the TTS changes with traction force and velocity pairs. The system model is the foundation to describe the train movement.

\subsection{System model}

The movement of the HST is driven by the interaction of traction or braking force, aerodynamic drag resistance $R(v)$ and the additional resistance $M g \sin (\alpha)$ caused by the gradients of the track. The train dynamic model is presented as eqns (1) and (2)

$$
\begin{gathered}
\frac{\mathrm{d} v}{\mathrm{~d} s}=\frac{F_{\mathrm{t}}-F_{\mathrm{b}}-R(v)-M g \sin (\alpha)}{M v}, \\
\frac{\mathrm{d} t}{\mathrm{~d} s}=\frac{1}{v}
\end{gathered}
$$

- $F_{\mathrm{t}}$ is the traction force.

- $F_{\mathrm{b}}$ is the braking force.

- $\quad v$ is the train speed.

- $s$ is the train position.

- $t$ is the train operation time.

- $\quad M$ is the train mass.

- $\quad \alpha$ is the angle of the gradient.

The calculation of $R(v)$ is given as eqn (3), which is based on field tests. 


$$
R(v)=M\left(a+b v+c v^{2}\right),
$$

- $\quad a, b$ and $c$ are constant values.

The traction power of the HST on traction motors $P_{\mathrm{w}}$ is calculated as

$$
P_{\mathrm{w}}=F_{\mathrm{t}} \cdot v \text {. }
$$

An accurate power consumption model should be introduced in this paper. The power of the rectifier $P_{\mathrm{t}}$ can be calculated as

$$
P_{\mathrm{t}}=\left\{\begin{array}{cc}
P_{\mathrm{w}}+P_{1} & \left(P_{\mathrm{w}}+P_{1}>0 \text { and } P_{\mathrm{w}} \neq 0\right) \\
0 & P_{\mathrm{w}}=0
\end{array},\right.
$$

- $\quad P_{1}$ is the power loss of the TTS.

The power losses of the TTS for different speed and traction force pairs are shown in Fig. 1.

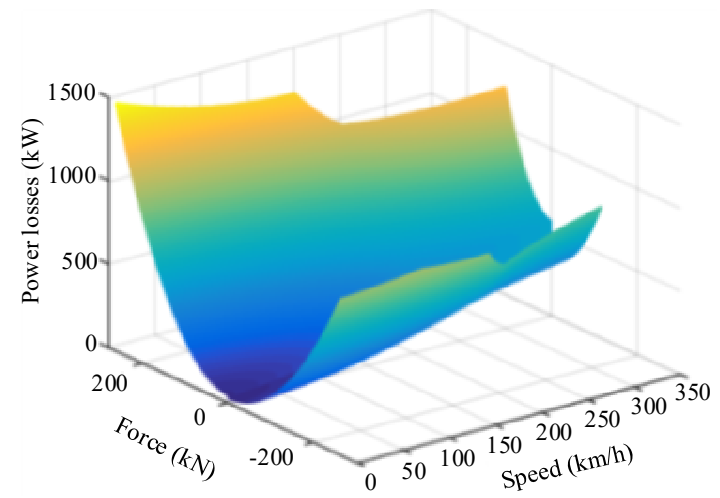

Figure 1: Power loss for the TTS.

\subsection{Optimization problem}

To minimize energy consumption, the cost function is written as

$$
\mathrm{J}=\int_{s_{0}}^{s_{f}} \frac{P_{\mathrm{t}}}{v} d s
$$

- $s_{0}$ and $s_{f}$ are the start and terminal position of the planned journey.

The operation constraints of the HST can be summarized as two groups: equality constraints and inequality constraints.

1. Equality constraints: the punctuality of railway is important, which can be regarded as

$$
\begin{gathered}
t\left(s_{f}\right)-t\left(s_{0}\right)=T, \\
v\left(s_{0}\right)=0 \text { and } v\left(s_{f}\right)=0,
\end{gathered}
$$

- $\quad T$ is the planned trip time. 
2. Inequality constraints: the control forces are bounded by the output characteristics of the TTS, expressed as

$$
\begin{aligned}
& 0 \leq F_{\mathrm{t}} \leq F_{\mathrm{t}, \max }(v), \\
& 0 \leq F_{\mathrm{b}} \leq F_{\mathrm{b}, \text { max }}(v) .
\end{aligned}
$$

In addition, the train speed cannot exceed the speed limitations of the track

$$
v \leq v_{\lim }(s)
$$

- $\quad F_{t, \max }(v)$ and $F_{b, \max }(v)$ are the maximum traction force and braking force, respectively.

- $v_{\text {lim }}(s)$ is the speed limitation of the track.

The optimization problem for minimizing the electrical energy consumption of the HST is a typical nonlinear programming problem, and the optimization method is illustrated in next section.

\section{OPTIMIZATION METHOD}

\subsection{Structure of optimization method}

For the optimization model with nonlinear power loss of the TTS, the optimized operation strategies can be found by dividing the problem into two subproblems, shown in Fig. 2.

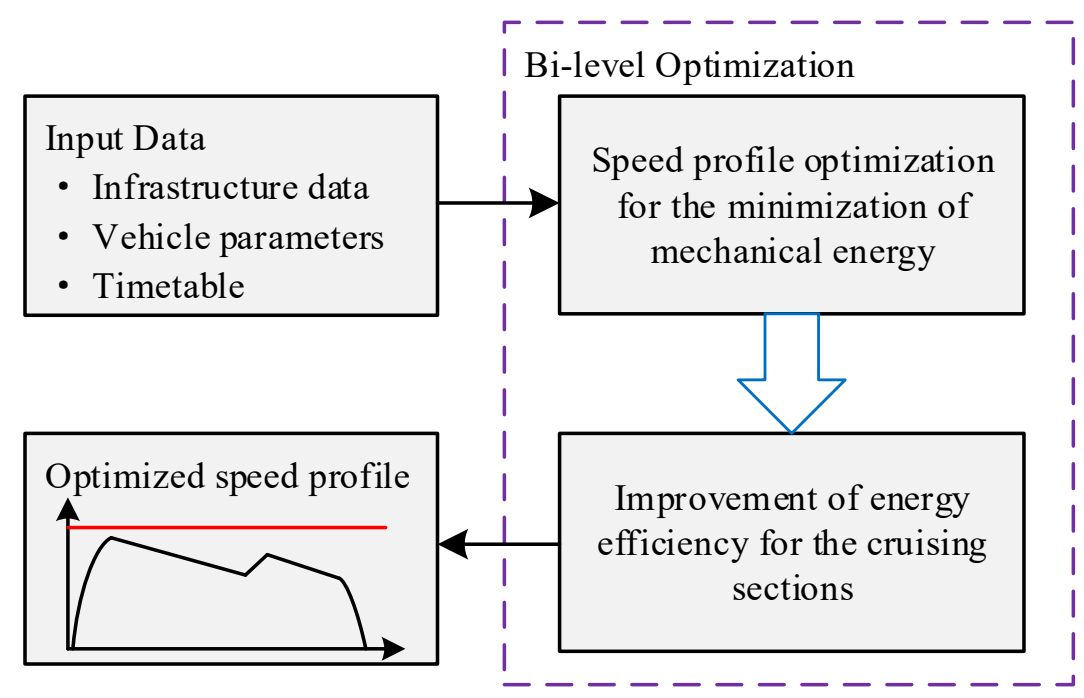

Figure 2: Structure of the bi-level optimization method.

For the optimization of mechanical energy, the planned timetable (i.e., the start and stop stations, the planned operation time), infrastructure data (i.e., the track gradients, curves and speed limits) and vehicle parameters (i.e., traction/braking characteristics, nonlinear power loss model and running resistance coefficient) are sent to the optimization subsystem. The PMP method is used to generate the optimal speed profile with minimal energy consumption on wheels. 
For the improvement of energy efficiency on the cruising sections, the cost function is the electric energy considering the nonlinear power loss of the TTS. In the cruising sections of the speed profile with minimization mechanical energy, the energy efficiency of the TTS might be low. The traction and coasting $(\mathrm{TnC})$ strategy is used to replace the cruising sections under the same operation time and distance constraints, which aims to improve the energy efficiency and reduce energy consumption.

3.2 Speed profile optimization with the minimization of mechanical energy

To minimize the mechanical energy, the cost function is written as

$$
\mathrm{J}^{\prime}=\int_{s_{0}}^{s_{f}} \frac{P_{\mathrm{w}}}{v} d s
$$

The optimal modes with minimum mechanical energy can be derived from the Maximum Principle [2], and the Hamiltonian function is defined as

$$
H=-\frac{P_{\mathrm{w}}}{v}+\frac{\lambda_{1}}{v}+\lambda_{2} \cdot \frac{F_{\mathrm{t}}-F_{\mathrm{b}}-R(v)-M g \sin (\alpha)}{M v} .
$$

The two adjoint variables $\lambda_{1}$ and $\lambda_{2}$ are determined by

$$
\begin{gathered}
\frac{d \lambda_{1}}{d s}=-\frac{\partial H}{\partial v}, \\
\frac{d \lambda_{2}}{d s}=-\frac{\partial H}{\partial t}=0 .
\end{gathered}
$$

To maximum the Hamiltonian function, the four optimal modes are:

- Full Traction $\left(F_{\mathrm{t}}=F_{\mathrm{t}, \max }(v)\right)$, which is limited by the maximum traction force.

- Cruising at constant speed

- Coasting

- Full Braking $\left(F_{\mathrm{b}}=F_{\mathrm{b}, \max }(v)\right)$, which is limited by the maximum deceleration.

The speed profile can be constructed based on these modes. The typical sequence is shown in Fig. 3. The key technologies are the calculation methods to determine the cruising speed $v_{\mathrm{c}}$ and the end speed $v_{\mathrm{b}}$ of coasting with the constraint of operation time.

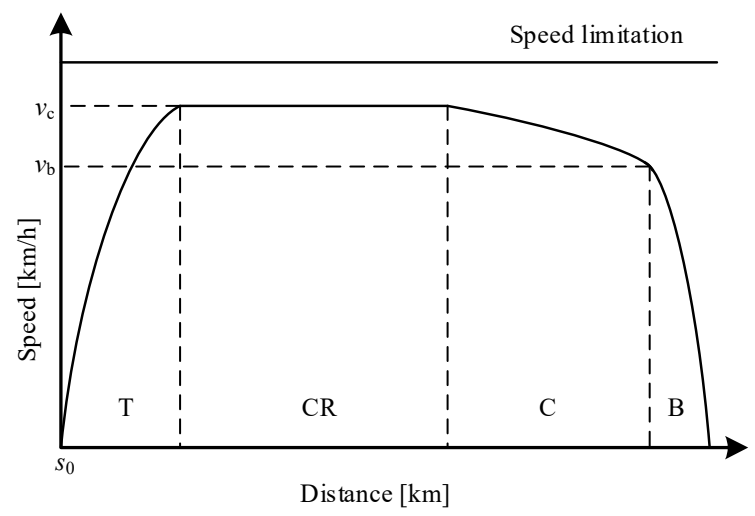

Figure 3: The typical optimal sequence for minimizing mechanical energy. 
When the coating phase is on the same slope values, the relationship of the two speed values can be derived based on eqns (1), (13) and (14)

$$
\begin{gathered}
v_{\mathrm{b}}=\frac{\psi\left(v_{\mathrm{c}}\right)}{R\left(v_{\mathrm{c}}\right)+\frac{\psi\left(v_{\mathrm{c}}\right)}{v_{\mathrm{c}}}-\sin \alpha}, \\
\psi\left(v_{\mathrm{c}}\right)=v_{\mathrm{c}}{ }^{2} R^{\prime}\left(v_{\mathrm{c}}\right) .
\end{gathered}
$$

By the iteration of cruising speed $v_{\mathrm{c}}$, the optimized speed profile can be obtained under the constraint of trip time.

\subsection{Improvement of energy efficiency on the cruising sections}

The cruising mode is the most energy saving strategy under the distance and time constraints when the cost function is the mechanical energy on wheels [2]. However, the load of the cruising mode is lower than the full traction, and the power loss of the TTS would increase. The TnC strategy is proposed to reduce the power loss on the TTS. The main idea of the TnC is to increase the load of the TTS by the full traction mode and improve the energy efficiency, and the coast mode does not consume energy. Two situations would occur when the TnC strategy is used, shown as Fig. 4(a) and Fig. 4(b).

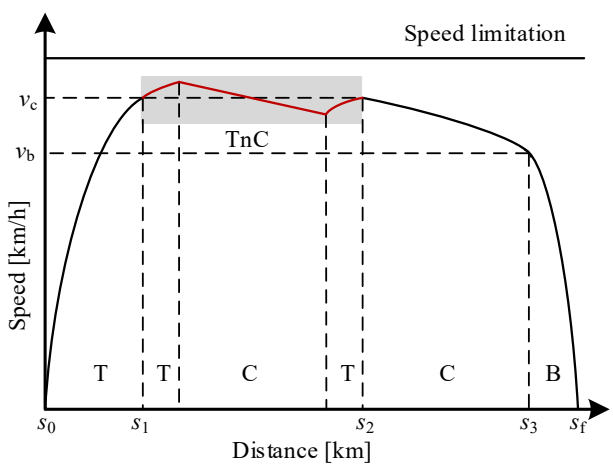

(a)

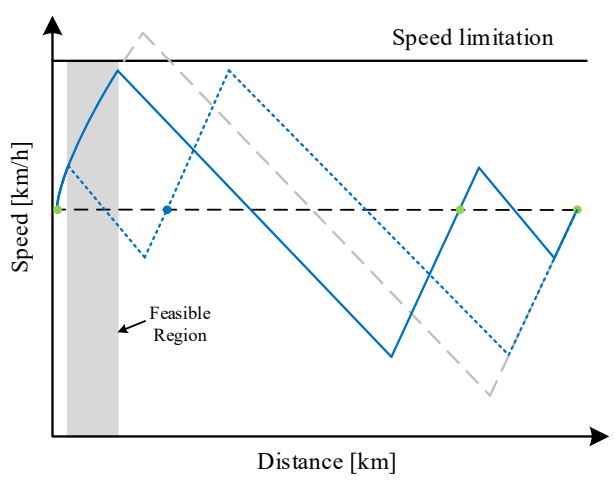

(b)

Figure 4: The TnC strategy for minimizing electrical energy.

In Fig. 4(a), the maximum speed of the TnC strategy is under the speed limitations, and one pair of traction and coast mode can replace the cruise mode under same time and distance constraints. In addition, the maximum speed of the TnC strategy might exceed the speed limitations when one pair of traction and coast mode is used, shown as the grey dotted line in Fig. 4(b). In this case, more TnC pairs should be utilized. Nowadays, the HSTs are mainly operated by drivers. To reduce the switching frequency of the operation mode, we should try to reduce the TnC pairs. Fig. 4(b) illustrated the feasible region determined by the two boundary linkage sequences as the green and blue lines. Then the energy-optimal operation strategy can be determined by the brute force search in the feasible region.

To summarize, the proposed method is shown as the following steps: 
1. Denote $v_{c}=\frac{s_{f}}{T}$, then the value of $v_{\mathrm{b}}$ is determined, and the optimized speed profile with the minimum mechanical energy can be obtained. Comparing the operation time $T_{\mathrm{c}}$ with the given trip time $T$. If $\left|T_{c}-T\right|<\varepsilon$, turn to step 2. Otherwise, adjust the speed $v_{c}$.

2. Find the start and end positions of the cruise mode, one pair of the TnC strategy is used to replace the cruise mode.

3. Compare the maximum speed of the $\mathrm{TnC}$ with speed limitations, if the maximum speed is lower than the speed limitation, the optimal speed profile is the strategy with smaller energy consumption, either the cruise strategy or the TnC strategy. if the maximum speed is larger than the speed limitation, turn to step 4.

4. Find the feasible region show in Fig. 4(b) using the multiple TnC pairs. In the feasible region, the energy-optimal strategy can be found by brute force search.

\section{QUANTITATIVE ANALYSIS}

Although the TnC strategy can reduce the energy loss on the TTS, the loss would increase caused by the fluctuation of speed profile. The quantitative analysis of the energy consumption for the $\mathrm{TnC}$ and the cruising strategies should be discussed. The load on the TTS has a great impact on the energy performance of the HST, which determines the energy-efficient operation strategy that is the cruising or the TnC. The constant speed of the cruising mode and the gradients of the track are two parameters to influence the TTS load.

\subsection{Train characteristics}

The CRH3 high-speed train is selected to evaluate the performance of the TnC and cruising strategies. The maximum traction force and aerodynamic drag resistance are presented in Fig. 5.

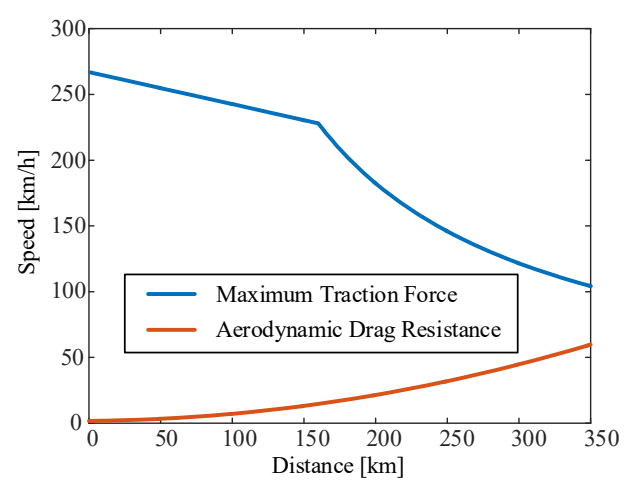

Figure 5: The maximum traction force and aerodynamic drag resistance.

\subsection{Analysis for different constant speed values}

The aerodynamic drag resistance is proportional to the square of the train speed, and it becomes the main energy consumer at high speed [7]. With the increase of the constant speed, the bigger traction force is required to overcoming the aerodynamic drag resistance, and the energy efficiency of the TTS would increase. The flat track is chosen in this case, and the range of investigated speed is from $150 \mathrm{~km} / \mathrm{h}$ to $300 \mathrm{~km} / \mathrm{h}$. 
Fig. 6(a) illustrates the speed profiles of the TnC and the cruising strategies at $150 \mathrm{~km} / \mathrm{h}$, $225 \mathrm{~km} / \mathrm{h}$ and $300 \mathrm{~km} / \mathrm{h}$. Moreover, the corresponding energy efficiency profiles are shown in Fig. 6(b). It can be observed that the coasting phase becomes shorter with the increase of constant speed values. The energy efficiency of the TnC strategy changes slightly at different constant speed, but the efficiency of the cruising strategy improves significantly when the constant speed values are increased.

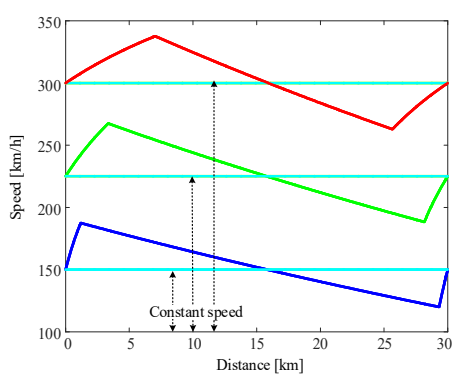

(a)

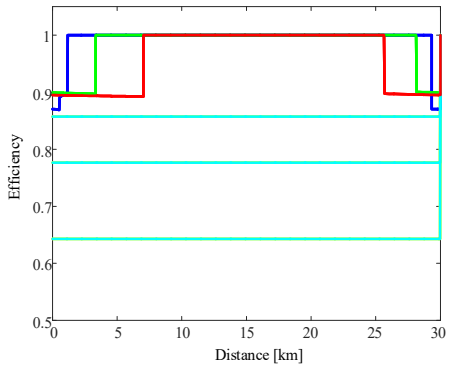

(b)

Figure 6: The results for different speed values. (a) Speed profile; and (b) Efficiency profile.

The energy consumption for different constant speed values is shown in Table 1. From the table, we can see that the more energy is needed to drive the HST either of the cruising mode or the $\mathrm{TnC}$ strategy when the constant speed value is risen. In addition, the TnC strategy can save energy under different constant speed values, and the energy saving rate is decreased with the increase of the constant speed value.

Table 1: Energy consumption for different constant speed values.

\begin{tabular}{|c|c|c|c|}
\hline $\begin{array}{c}\text { Constant value } \\
(\mathrm{km} / \mathrm{h})\end{array}$ & $\begin{array}{c}\text { Cruising mode } \\
(\mathrm{kW} \cdot \mathrm{h})\end{array}$ & $\begin{array}{c}\text { The TnC } \\
(\mathrm{kW} \cdot \mathrm{h})\end{array}$ & $\begin{array}{c}\text { Energy saving } \\
\text { rate }(\%)\end{array}$ \\
\hline 150 & 175.1973 & 133.4892 & 23.81 \\
\hline 175 & 212.2834 & 169.7276 & 20.05 \\
\hline 200 & 251.6512 & 211.0614 & 16.13 \\
\hline 225 & 291.0044 & 258.5505 & 11.15 \\
\hline 250 & 336.5951 & 311.8014 & 7.37 \\
\hline 275 & 388.3206 & 370.269 & 4.65 \\
\hline 300 & 446.2018 & 433.8835 & 2.76 \\
\hline
\end{tabular}

\subsection{Analysis for different gradients}

The gradient of the track is another important factor that influences the load of the train at cruising mode. The energy values for different constant speed and gradients pairs are presented in Fig. 7. When the cruise mode occurs on the track with slope less than $2 \%$, the TnC strategy can save energy under different constant speed values, and the energy saving rate decreases with the increase of the TTS load. The energy consumption of the TnC is larger than the cruising mode when the speed is $150 \mathrm{~km} / \mathrm{h}$ and the gradient is $6 \%$. This indicates that the fluctuation of speed will increase the energy loss caused by aerodynamic drag resistance, although the power loss on the TTS is low using the TnC. 


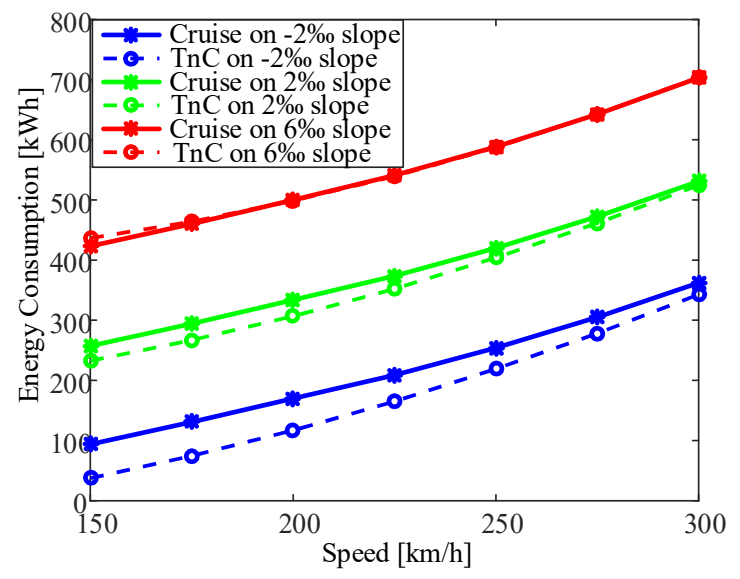

Figure 7: The energy consumption for different constant speed and gradients.

\section{CASE STUDY}

The distance of the simulation high-speed railway line is $70 \mathrm{~km}$, and the planned trip time is $1200 \mathrm{~s}$. The characteristics of the HST are shown in Fig. 5. Two cases are designed to evaluate the proposed TnC strategy.

- Case 1: the speed limitation is not considered, which means one pair of the TnC can replace the cruising mode.

- Case 2: the speed limitation is considered, and two pairs of the TnC should be adopted.

\subsection{Case 1}

This case aims to evaluate the effectiveness of the TnC strategy without considering the speed limitations. Firstly, the speed profile with the cruising mode based on the PMP is obtained in Fig. 8(a), and then the TnC strategy is used to replace the cruise mode. The energy efficiency profiles of the two strategies are shown in Fig. 8(b).

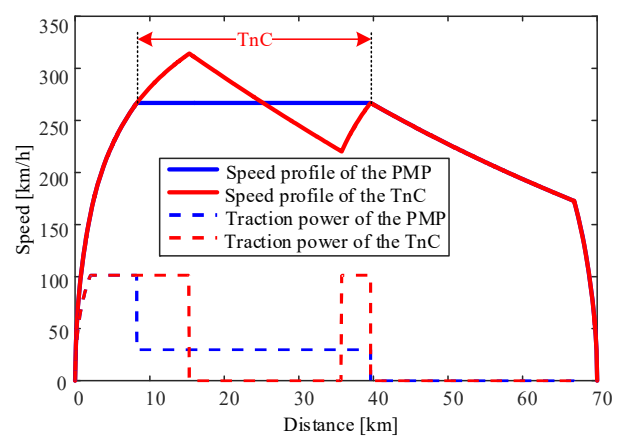

(a)

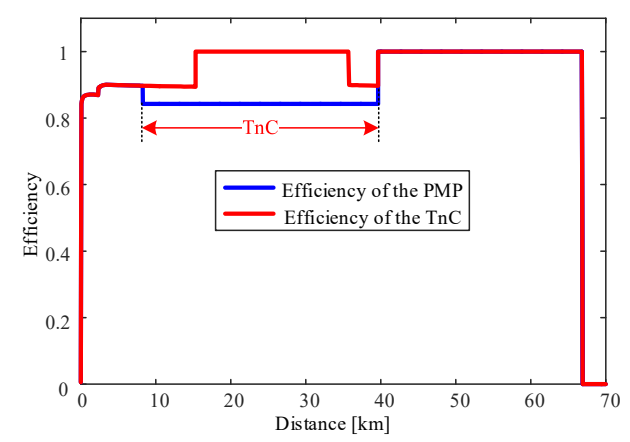

(b)

Figure 8: The speed profiles and energy efficiency. (a) Speed profile; and (b) Energy efficiency. 
The cruising speed obtained by the PMP is $266.8 \mathrm{~km} / \mathrm{h}$, and the energy efficiency of the cruising mode is 0.8425 . The maximum speed of the TnC strategy is $314 \mathrm{~km} / \mathrm{h}$, and the energy efficiency of the $\mathrm{TnC}$ is improved significantly. For the $\mathrm{TnC}$ operation section, the electrical energy consumption of the PMP and the TnC are $474.8873 \mathrm{kWh}$ and $462.7282 \mathrm{kWh}$, respectively. The energy saving rate is $2.56 \%$ of the TnC strategy.

Table 2 illustrates the energy loss caused by the basic resistance and the TTS. It shows that the reduced loss of the TTS is larger than the increased energy consumption of the basic resistance, and the energy efficiency can be improved by the TnC strategy when the nonlinear power loss model of the TTS is considered.

Table 2: Energy consumption for different devices.

\begin{tabular}{|c|c|c|}
\hline $\begin{array}{c}\text { Operation } \\
\text { strategy }\end{array}$ & $\begin{array}{c}\text { Energy loss of resistance } \\
(\mathrm{kW} \cdot \mathrm{h})\end{array}$ & $\begin{array}{c}\text { Energy loss of the TTS } \\
(\mathrm{kW} \cdot \mathrm{h})\end{array}$ \\
\hline The TnC & 404.5647 & 58.1635 \\
\hline The Cruising & 395.7458 & 79.1415 \\
\hline
\end{tabular}

\subsection{Case 2}

This case aims to find the switching sequence of the multiple TnC pairs when the maximum traction speed of one TnC pair exceeds the speed limitations. The speed profiles of different switching positions are shown in Fig. 9(a). The operation time and distance for theses profiles are the same. Furthermore, the relationship between the energy consumption and the switching position of the first coast mode is shown in Fig. 9(b). It can be observed that the energy consumption decreases with the increase of the position before $3.19 \mathrm{~km}$, and then the energy consumption increases with the position. A minimum energy consumption can be found, and the energy-optimal switching sequence of multiple $\mathrm{TnC}$ pairs can be determined.

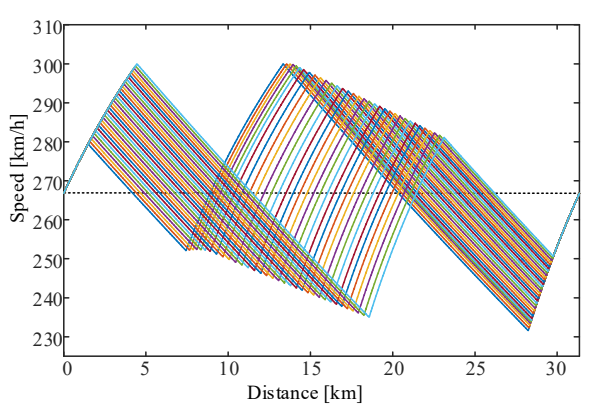

(a)

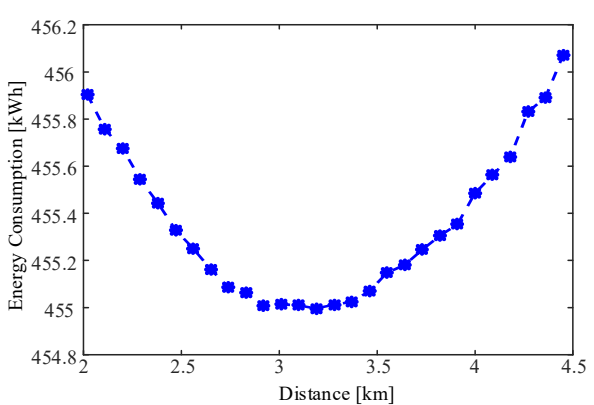

(b)

Figure 9: The performance of different TnC pairs switching position. (a) Speed profile; and (b) Energy consumption

In this case, the speed profile based on the PMP is the same as Fig. 8(a). The strategy with two pairs TnC is shown in Fig. 10(a), and the related energy efficiency profiles are shown in Fig. 10(b). The maximum speed of the TnC strategy is $289 \mathrm{~km} / \mathrm{h}$, and the energy consumption of the TnC is $455 \mathrm{kWh}$. The two pairs TnC strategy can reduce electrical energy 
consumption further compared to the one pair TnC strategy. It is obvious that the two pairs $\mathrm{TnC}$ strategy is more complex than the only one pair TnC strategy for drivers.

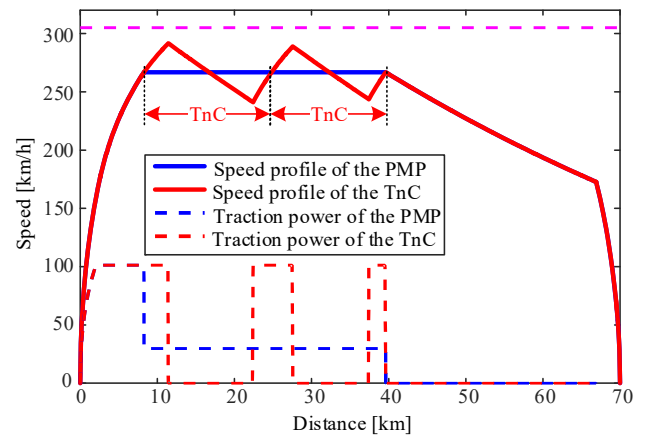

(a)

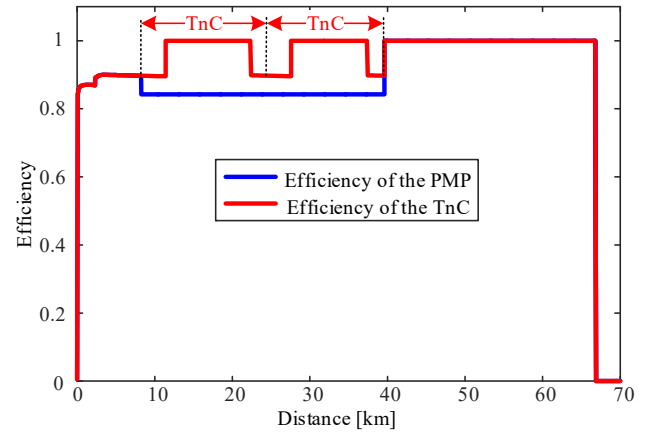

(b)

Figure 10: The speed profiles and energy efficiency. (a) Speed profile (b) Energy efficiency.

\section{CONCLUSION}

This paper presents a bi-level optimization method to minimize the electrical energy consumption with the nonlinear power loss model of the TTS for the HSTs. Firstly, the minimum mechanical energy on wheel is obtained based on the PMP, and the speed profile is constructed with four optimal modes such as full traction, cruising, coasting and full braking. Then, the TnC driving strategy is proposed to improve the energy efficiency of the TTS when the train is in cruising mode with low load. Comparing optimized results with the solution based on cruise control, the energy saving rate can be achieved without and with speed limitations. In addition, the performance of proposed TnC strategy is illustrated and analyzed under different constant speed values and gradients. For application, the proposed method can be used for a driver assistant system (DAS) or an automatic train operation (ATO) system to improve the energy economy of the high-speed railway.

\section{ACKNOWLEDGEMENT}

This research was supported by the National Key Research and Development Program of China under the Grant 2017YFB1201302 and Grant 2016YFB1200502.

\section{REFERENCES}

[1] Wang, K., Hu, H., Chen, J., Zhu, J., Zhong, X. \& He, Z., System-level dynamic energy consumption evaluation for high-speed railway. IEEE Transactions on Transportation Electrification, 5(3), pp. 745-757, 2019.

[2] Howlett, P.G., Milroy, I.P. \& Pudney, P.J., Energy-efficient train control. Control Engineering Practice, 2(2), pp. 193-200, 1994.

[3] Zhao, X.-H., Ke, B.-R. \& Lian, K.-L., Optimization of train speed curve for energy saving using efficient and accurate electric traction models on the mass rapid transit system. IEEE Transactions on Transportation Electrification, 4(4), pp. 922-935, 2018.

[4] Yang, X., Li, X., Ning, B. \& Tang, T., A survey on energy-efficient train operation for urban rail transit. IEEE Transactions on Intelligent Transportation Systems, 17(1), pp. $2-13,2016$. 
[5] Scheepmaker, G.M., Goverde, R.M. \& Kroon, L.G., Review of energy-efficient train control and timetabling. European Journal of Operational Research, 257(2), pp. 355376, 2017.

[6] Tian, Z., Zhao, N., Hillmansen, S., Roberts, C., Dowens, T. \& Kerr, C., Smartdrive: Traction energy optimization and applications in rail systems. IEEE Transactions on Intelligent Transportation Systems, 20(7), pp. 2764-2773, 2019.

[7] Xiao, Z., Feng, X., Wang, Q. \& Sun, P., Eco-driving control for hybrid electric trams on a signalized route. IET Intelligent Transport Systems, 14(1), pp. 36-44, 2020.

[8] Wong, K.K. \& Ho, T.K., Dynamic coast control of train movement with genetic algorithm. International Journal of Systems Science, 35(13), pp. 835-846, 2004.

[9] Wang, Y., De Schutter, B., van den Boom, T.J. \& Ning, B., Optimal trajectory planning for trains-a pseudospectral method and a mixed integer linear programming approach. Transportation Research Part C: Emerging Technology, 29, pp. 97-114, 2013.

[10] Albrecht, A., Howlett, P., Pudney, P., Vu, X. \& Zhou, P., The key principles of optimal train control-part 1: Formulation of the model, strategies of optimal type, evolutionary lines, location of optimal switching points. Transportation Research Part B: Methodologies, 94, pp. 482-508, 2016.

[11] Albrecht, A., Howlett, P., Pudney, P., Vu, X. \& Zhou, P., The key principles of optimal train control - Part 2: Existence of an optimal strategy, the local energy minimization principle, uniqueness, computational techniques. Transportation Research Part B: Methodologies, 94, pp. 482-508, 2016.

[12] Liu, R.R. \& Golovitcher, I.M., Energy-efficient operation of rail vehicles. Transportation Research Part A: Policy Practice, 37(10), pp. 917-932, 2003.

[13] Frank, P.T. \& Meyer, M., An algorithm for the optimal control of the driving of trains. Proceedings of the 39th IEEE Conference on Decision and Control, pp. 2123-2127, 2000. 